\title{
Social Network Sites Use and Psychological distress: A Systematic Review
}

\author{
Andrew Samy Helmy Hanna \\ Faculty of Medicine \\ Ain Shams University Cairo Egypt \\ androsami0100054@med.asu.edu.eg
}

\author{
Eila Erfani \\ Faculty of Engineering and \\ Information Technology \\ University of Technology Sydney \\ Eila.erfani@uts.edu.au
}

\author{
Layla Boroon \\ Faculty of Engineering and \\ Information Technology \\ University of Technology Sydney \\ undefined layla.boroon@uts.edu.au
}

\begin{abstract}
Social Network Sites (SNSS) have become a pervasive part of people's everyday lives, the implications of their use need to be investigated and understood. We conducted a systematic literature review to lay the groundwork for understanding the relationship between SNS use and psychological distress. The review included articles published between 2003 and 2020 , extracted from major academic databases. Findings revealed that the use of SNSs use can be associated to psychological distress. This study shows the factors such as depressive rumination, narcissistic behavior, excessive and unsupervised SNS use that mediate this relationship.
\end{abstract}

Keywords: Systematic literature review, social network sites, psychological distress.

\section{Introduction}

Social Network Sites (SNSs) are "web-based communication platforms with three distinct characteristics: (1) user profiles are unique and created through user-provided content and content provided by other users, (2) the network connections between individuals are visible and can be navigated through by other users, and (3) individuals can broadcast content and consume and interact with content contributed by others in a continuous stream of information" [1]. The explosive growth of SNSs has made SNS use and its consequences increasingly popular topics for research. SNS could be regarded as a 'double-edged sword'.

Studies have investigated the positive and negative impact of SNSs use on users' health outcome [2], however considerable disagreement exists about the impact of SNS use on users' mental health. While some studies have shown SNSs enable users to obtain, informational, emotional, network and esteem support and consequently experience better mental health and psychological wellbeing [3,4], others have shown miscommunications and maladaptive behaviors on SNS can negatively impact users' mental health and lead to psychological distress in terms of anxiety, depression and loneliness [5].

Understanding the impact of SNS users on users has become a priority due to a simultaneous increase in mental health problems [6]. As SNS use has become part of people's daily activities introducing the potential positive and negative impact of SNS use on users' mental health would be a major advance.

A recent systematic literature review have been conducted to lay the groundwork for understanding the relationship between the use of SNSs and users' psychological well-being [2]. However, our understanding of the relationship between the use of SNSs and users' psychological distress remains partial [6].

Psychological distress is a general term used to describe unpleasant feelings or emotions that impact individual's level of functioning. In other words, it is psychological discomfort that interferes with activities of daily living. Depression, anxiety, loneliness and sadness are manifestations of psychological distress [7]. Psychological distress is a subjective experience. That is, the severity of psychological distress is dependent upon the situation and how we perceive it. We can think of psychological distress as a continuum with 'mental health' and 'mental illness' at opposing ends. Psychological distress can be thought of as a maladaptive response to a stressful situation and occurs when external events or stressors place demands upon us that we are unable to cope with [7]. This study considered depression, anxiety, loneliness and sadness as indexes of psychological distress.

The research presented in this paper aimed to determine what is already known about the relationship between the use of SNSs and 
psychological distress of users, synthesize the current research evidence, inform policy and practice and propose an agenda for future studies on this topic. In particular, our systematic literature review was designed to answer the question: (i) What SNS platforms have been studied? (ii) How SNSs and users' psychological distress were measured? (iii) What are the mediators of the relationship between SNS use and psychological distress?

The remainder of this article is organized as follows. Section two describes our methods, results are outlined in section three, section four contains recommendations for future studies, and our conclusions are presented in section five.

\section{Method}

Standard Preferred Reporting Items for Systematic Reviews and Meta-Analyses (PRISMA) guidelines were used to guide our systematic review of relevant peer-reviewed literature [8].

Eligibility criteria. We applied a set of inclusion and exclusion criteria for identifying studies that provide direct and clear evidence about the relationship between the use of SNSs and users' psychological distress. To reduce the likelihood of bias, selection criteria were identified on the basis of our research questions (see Introduction section). The inclusion criteria were that papers must be: (i) published in peer-reviewed journals, (ii) written in English, (iii) published between 2003 and 2020, and (iv) related to the research questions. Exclusion criteria ensured that selected articles would not be: (i) only focused to the context of general psychological distress (or similar), (ii) studies limited to general use of the Internet or Web 2.0 applications other than SNSs, such as content-oriented sites (e.g., Twitter) and (iii) non-academic studies .

Search strategy In February 2019 a keyword search spanning the period between 2003 and 2020 was executed in major databases (Scopus, Web of Science, PsycInfo and HMIC) to capture published research related to SNSs and psychological distress. Our search period started from 2003 as major SNSs were launched after this date. In addition.

We also looked at controlled vocabularies (Subject heading/Thesaurus) in databases for more complete search. Derived from some prior studies, the combination of terms ("Social Network SiteS" OR "Online Social Network SiteS" OR "Social media" OR "Social Networking SiteS”) AND ("psychological distress" OR "anxiety" OR “depression") AND
((“01/02/203”: “01/02/2020”)) AND ((English [lang])) was used to search titles and abstracts.

Scopus and Web of Science were used as they are multidisciplinary databases and cover all the subject areas. PsycInfo and HMIC were used because they cover specialized studies in psychology and behavioural sciences as well as health service policy, management, and administration disciplines. Furthermore, we included major conference proceedings as well as Information Systems journals.

Study Selection The search identified 340 articles; the removal of duplicates left 156. Papers' titles, abstracts and keywords were screened by both authors, which resulted in the exclusion of 94 articles which were not clearly pertinent: they did not match the research questions, and were focused on general internet use. Both authors assessed the 62 remaining independently and identified potentially relevant papers, with any disagreements resolved by discussion. In all, 33articles were excluded for the following reasons: no focus on SNSs (e.g., focus was on blogs or micro- blogging sites like Twitter), no focus on psychological distress in terms of anxiety and depression, and loneliness and being basic or laboratory science. This process resulted in 29 papers for final assessment (Appendix 1). Figure 1 illustrates the procedure of literature search and selection.

We measured the reliability of agreement between ourselves with Krippendorff's alpha (Krippendorff, 2004), a well-suited measure of reliability in content analysis. A Krippendorff's alpha of 0.890 was achieved, reflecting high reliability of agreement (this value should be 0.800 or higher)[9]. Next, each article was independently assessed by both authors for quality assessment. Some of the criteria for quantitative studies:

- Analytic methods described/justified and appropriate?

- Some estimate of variance is reported for the main results?

- Controlled for confounding?

- Method of subject/comparison group selection or source of information/input variables described and appropriate?

- Outcome and (if applicable) exposure measure(s) well defined and robust to measurement/misclassification bias?

- Means of assessment reported?

- Some estimate of variance is reported for the main results?

Criteria for qualitative studies were as following:

- Study design evident and appropriate? 
- Context for the study clear?

- Connection to a theoretical framework/wider body of knowledge?

- Sampling strategy described, relevant and justified?

- Data collection methods clearly described and systematic?

- Data analysis clearly described and systematic?

- Use of verification procedure(s) to establish credibility?

Data Extraction and Data Synthesis. The objective of this step was to synthesize the information obtained from the selected studies and summarize the results. To this end, all 29 articles were imported to NVivo for qualitative analysis. We selected NVivo for conducting our literature review because it maximizes precision with minimal effort, reduces reliance on memory and misplacing of important data, facilitates data handling and review, and enables rapid identification of co-occurring references in articles. We then coded the data extracted from each paper independently, and resolved disagreements by discussion. Data synthesis employed a narrative approach - a descriptive qualitative approach that is widely used in the synthesis of heterogeneous studies [9]. We analyzed the extracted information with respect to our research questions.

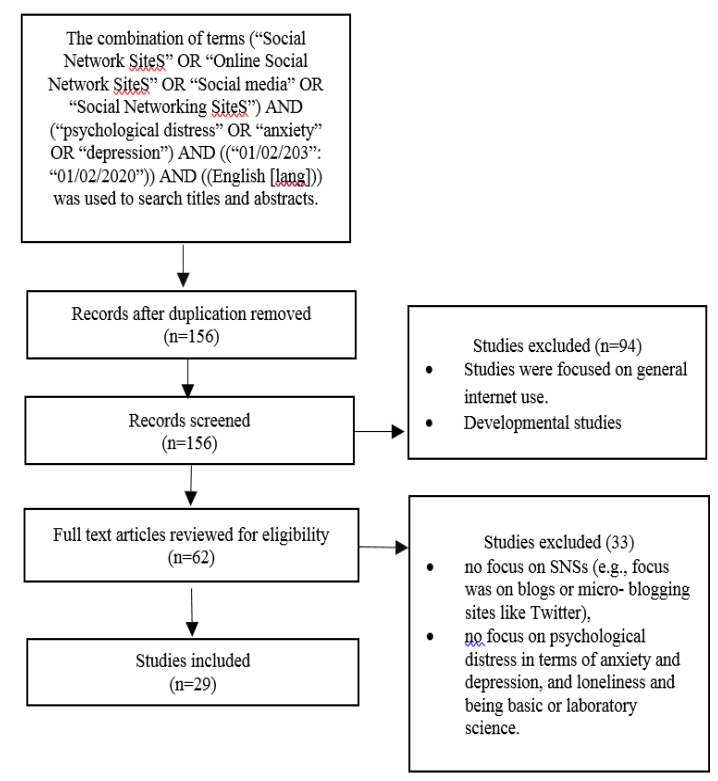

FEG 1. Flowchart of the systematic literature search

\section{Results}

Thematic analysis, the process of collecting candidate themes and creating relationships between these themes, was used to identify, analyze, and report themes found in the reviewed papers. We analyzed the under review papers using NVivo 8, a software that facilitates the coding and sorting process [9]. Through six phases: familiarization with the data, generating initial codes, searching for themes, reviewing themes, defining and naming themes, and producing the final reports. First, each paper was uploaded to NVivo and read several times, to obtain a sense of the entire paper. Content areas were developed based on theoretical assumptions derived from the literature. Within each content area, the text was divided into meaning units. The condensed meaning units were abstracted and labelled with a code. The various codes were compared and sorted into nodes in NVivo. Codes that were closely linked in meaning were formed into categories, creating the manifest content. Next, the underlying meanings - that is, the latent content - of these categories were formulated into themes. Themes were reviewed to compare and reconcile discrepancies, and themes with a similar meaning were combined in matching nodes.

\section{What types of SNS platforms have been studied?}

As table 1 (supplementary file 1) shows Facebook was the most popular SNS platform studied, while the remaining studies investigated one or more other platform, neglecting other cohorts of SNS users and their patterns of use and needs. Just two studies investigated MySpace (FB/MS), instant messaging (IMing; e.g., online instant messaging such as AOL IM, Microsoft Messenger, Google Talk, Yahoo! Messenger, iChat, ICQ, Skype, or eBuddy).

\section{How SNS use and users' psychological distress were measured?}

SNS use was measured in terms of intensity of use that refers to the extent of user engagement in SNS activities and amount of time spent on the network on a typical day, frequency of use that refers to the number of times per unit of time that users use SNS, history of use - how long (years and months) people have been a member of an SNS, type of use either passive or active users, purpose of use (social purpose or nonsocial purpose), size and structure of the SNS and quality of interactions(supportive or rumination). As table 1 (supplementary file 1) shows time spent on 
SNS and frequency of use were the most popular indexes for measuring SNS use.

Studies have measured psychological distress in terms depression, anxiety and loneliness. Depression refers to feelings of sadness and/or a loss of interest in activities. Anxiety refers to fear that are strong enough to interfere with one's daily activities and loneliness which is related to feeling sad and unhappy about being socially isolated. As table 1 (supplementary file 1) shows depression was the most popular measurement index of psychological distress used by most of studies.

\section{What are the mediators of the relationship between SNS use and psychological distress?}

Reviewed studies have shown the poor and negative quality of communications conducted in SNS is associated with experiencing psychological distress. Depressive rumination, narcissistic behavior patterns, and unmanaged use is also related to experiencing depression and anxiety. SNS use for entertainment contributes positively in experiencing higher level of loneliness. Figure 2 shows factors that can mediate the impact of SNS use on psychological distress.

- Depressive rumination: reviewed studies have shown that disruptive and negative interactions in SNS is related to experiencing psychological distress in terms of depression, anxiety and loneliness. There are many opportunities that can enhance miscommunications and mishandled expectations in SNS environment. Depressive rumination, a response style where an individual repetitive focus on their pain and negative interactions, in SNS are linked to growth and maintenance of anxiety and depression.

- Narcissistic behaviour: SNS use can also be associated with drop in one's self-esteem and increase of narcissistic behavior and consequently experiencing anxiety and depression.

People use SNS for various purposes, sometimes users tend to overstate their personal, professional and other qualities while at the same time hiding their potential faults. In some situations, SNS compare others and perceives other users are happier and more successful than themselves and this can lead to jealously and consequently to higher level of psychological distress.

In social networking setting, people tend to exaggerate their qualities and their happiness while at the same time hiding their potential faults and this may be one of the factors affecting mood in online users and be associated to experiencing depression and anxiety.

- Excessive and unsupervised SNS use: unmanaged, unsupervised time and excessive amount of tine spent on SNS use, and addictive online behavior use can reduce the available time for conventional face-to face interaction which can lead to loneliness. Unmanaged SNS use can lead to experience of negative social communications (e.g. cyberbullying), and in turn feeling higher level of depression and anxiety.

- SNS use for entertainment: studies showed that SNS use for nonsocial purpose and entertainment was related too higher level of loneliness and consequently psychological distress.

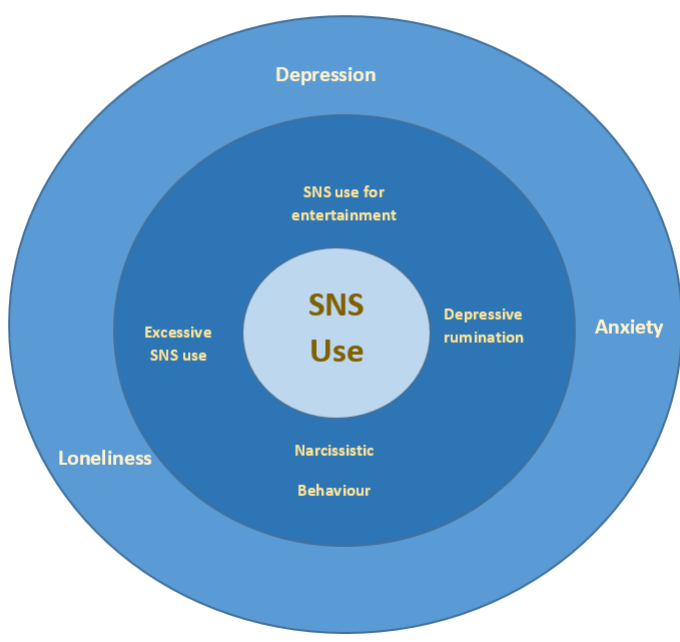

FEG 2. SNS use and psychological distress

\section{Recommendations for future studies}

Most of the studies have focused on healthy population samples, such as university students, adolescents, and school children. Future studies are therefore encouraged to consider other groups of SNS users, such as elderly, people with reduced mobility and/or those living with serious health conditions and chronic disease, people in vulnerable situations as well as people living in remote geographical areas.

There was not a strong theoretical background in any of the reviewed studies. Strong theoretical foundations are needed to explain negative consequences of SNS use as well as factors that can 
moderate the negative outcome. In addition the reviewed studies haven't shown the long-term impacts of the use of SNSs on individuals' psychological distress. Future studies are encouraged to employ longitudinal approaches for demonstrating the long-term effects of SNSs use. It is recommended that future empirical studies should be conducted to longitudinally examine the relationship between SNS use and factors that moderates the impact of SNS use users' psychological distress.

As most of studies investigated Facebook as SNS future research should also examine whether these findings generalize to other online social network.

\section{Conclusion}

This systematic review examined the recent research on associations between SNSs and psychological distress. The results of the study contribute to understanding of the circumstances that make the use of social networking to higher level of psychological distress including negative interaction, excessive use, and unsupervised use. Implications for understanding circumstances that increase social networking, as well as resulting negative interactions and negative affect are discussed. This study presents some factors that mediate the relationship between SNS use and experiencing psychological distress in SNS environment. It shows why some people are placed at risk once they use SNS, and enable users to address the detriments the use of SNSs may have on their mental health. This is important because, as alluded to earlier, the public has been very interested in whether social networking can be a breeding ground for depression. This study can inform policy makers and practice of the factors that mediates relationship between SNS use and psychological distress and will help them to develop strategies to reduce psychological stress experienced in SNS.

\section{References}

[1] Ellison, Nicole B., and Danah Boyd. "Sociality through social network sites." The Oxford handbook of internet studies (2013): 151-172.

[2] Erfani, Seyedezahra Shadi, and Babak Abedin. "Impacts of the use of social network sites on users' psychological well-being: A systematic review." Journal of the Association for Information Science and Technology 69, no. 7 (2018): 900-912.

[3] Guo, Yu, Yiwei Li, and Naoya Ito. "Exploring the predicted effect of social networking site use on perceived social capital and psychological well-being of Chinese international students in Japan." Cyberpsychology, Behavior, and Social Networking 17, no. 1 (2014): 52-58.

[4] Nabi, Robin L., Abby Prestin, and Jiyeon So. "Facebook friends with (health) benefits? Exploring social network site use and perceptions of social support, stress, and well-being." Cyberpsychology, Behavior, and Social Networking 16, no. 10 (2013): 721-727.

[5] Kalpidou, Maria, Dan Costin, and Jessica Morris. "The relationship between Facebook and the well-being of undergraduate college students." CyberPsychology, behavior, and social networking 14, no. 4 (2011): 183 189

[5] Kalpidou, Maria, Dan Costin, and Jessica Morris. "The relationship between Facebook and the well-being of undergraduate college students." CyberPsychology, behavior, and social networking 14, no. 4 (2011): 183 189

[6] Erfani, Seyedezahra Shadi, Babak Abedin, and Yvette Blount. "The effect of social network site use on the psychological well-being of cancer patients." Journal of the Association for Information Science and Technology 68, no. 5 (2017): 1308-1322.

[7] Winefield, H. R., Gill, T. K., Taylor, A. W., \& Pilkington, R. M. (2012). Psychological wellbeing and psychological distress: is it necessary to measure both? Psychology of WellBeing, 2(1), 1-14.

[8] Moher D, Liberati A, Tetzlaff J, Altman DG, The PRISMA Group (2009) Preferred Reporting Items for Systematic Reviews and MetaAnalyses: The PRISMA Statement. PLoS Med 6(7): e1000097. doi:10.1371/journal.pmed.1000097

[9] Erfani, Seyedezahra Shadi, and Babak Abedin. "Effects of Web based cancer support resources use on cancer affected people: A systematic literature review." The International Technology Management Review 4, no. 4 (2014): 201-211.

[10] Banjanin N, Banjanin N, Dimitrijevic I, Pantic I. Relationship between internet use and depression: focus on physiological mood oscillations, social networking and online addictive behavior. Comput Human Behav 2015 Feb;43:308-312.

[11] Davila J, Hershenberg R, Feinstein BA, Gorman K, Bhatia V, Starr LR. Frequency and quality of social networking among young adults: associations with depressive symptoms, rumination, and corumination. Psychol Pop Media Cult 2012 Apr 1;1(2):72-86

[12] Farahani HA, Kazemi Z, Aghamohamadi S, Bakhtiarvand F, Ansari M. Examining mental health indices in students using Facebook in Iran. Procedia Soc Behav Sci 2011;28:811-814.

[13] Feinstein BA, Bhatia V, Hershenberg R, Davila J. Another venue for problematic interpersonal behavior: the effects of depressive and anxious symptoms on social networking experiences. J Soc Clin Psychol 2012 Apr;31(4):356-382

[14] Jelenchick LA, Eickhoff JC, Moreno MA. "Facebook depression?" Social networking site use and depression in older adolescents. J Adolesc Health 2013 Jan;52(1):128-130 
[15]Locatelli SM, Kluwe K, Bryant FB. Facebook use and the tendency to ruminate among college students: testing mediational hypotheses. J Educ Comput Res 2012 Sep 7;46(4):377-394. [CrossRef]

[16]TaLup K, Trub L, Rosenthal L. Instagram \#instasad?: exploring associations among instagram use, depressive symptoms, negative social comparison, and strangers followed. Cyberpsychol Behav Soc Netw 2015 May; 18(5):247-252

[17]Pantic I, Damjanovic A, Todorovic J, Topalovic D, Bojovic-Jovic D, Ristic S, et al. Association between online social networking and depression in high school students: behavioral physiology viewpoint. Psychiatr Danub 2012 Mar;24(1):90-93

[18]Rae JR, Lonborg SD. Do motivations for using Facebook moderate the association between Facebook use and psychological well-being? Front Psychol 2015;6:771

[19]Shaw AM, Timpano KR, Tran TB, Joormann J. Correlates of Facebook usage patterns: the relationship between passive Facebook use, social anxiety symptoms, and brooding. Comput Hum Behav 2015 Jul;48:575-580.

[20]Simoncic TE, Kuhlman KR, Vargas I, Houchins S, Lopez-Duran NL. Facebook use and depressive symptomatology: investigating the role of neuroticism and extraversion in youth. Comput Hum Behav 2014 Nov;40:1-5.

[21]Steers MN, Wickham RE, Acitelli LK. Seeing everyone else's highlight reels: how Facebook usage is linked to depressive symptoms. J Soc Clin Psychol 2014 Oct;33(8):701-73

[22]Tandoc EC, Ferrucci P, Duffy M. Facebook use, envy, and depression among college students: is

Facebooking depressing? Comput Hum Behav 2015 Feb;43:139-146.

[24] E, Subrahmanyam K, Eggermont S. The short-term longitudinal and reciprocal relationships between peer victimization on Facebook and adolescents' wellbeing. J Youth Adolesc 2016 Sep;45(9):1755-1771. [CrossRef] [Medline]

[25] Weidman AC, Levinson CA. I'm still socially anxious online: offline relationship impairment characterizing social anxiety manifests and is accurately perceived in online social networking profiles. Comput Hum Behav 2015 Aug;49:12-19. [CrossRef]

[26] Park S, Lee SW, Kwak J, Cha M, Jeong B. Activities on Facebook reveal the depressive state of users. J Med Internet Res 2013;15(10):e217

[27] Park S, Kim I, Lee S, Yoo J, Jeong B, Cha M. Manifestation of depression and loneliness on social networks: a case study of young adults on Facebook. 2015 Presented at: Proceedings of the 18th ACM Conference on Computer-Supported Cooperative Work and Social Computing; 2015; Vancouver, Canada p. 557-570.

[28]Moreno MA, Jelenchick LA, Egan KG, Cox E, Young $\mathrm{H}$, Gannon KE, et al. Feeling bad on Facebook: depression disclosures by college students on a social networking site. Depress Anxiety 2011 Jun;28(6):447455
[29]Settanni M, Marengo D. Sharing feelings online: studying emotional well-being via automated text analysis of Facebook posts. Front Psychol 2015;6:1045

[30]Tsugawa S, Kikuchi Y, Kishino F, Nakajima K, Itoh Y, Ohsaki H. Recognising depression from Twitter activity. 2015 Presented at: Proceedings from the 33rd ACM Conference on Human Factors in Computing Systems; 2015; Seoul, Korea p. 3187-3196.

[31]Dumitrache SD, Mitrofan L, Petrov Z. Self-image and depressive tendencies among adolescent Facebook users. Revista de Psihologie 2012;58(4):285-295.

[32]Deters FG, Mehl MR, Eid M. Social responses to Facebook status updates: the role of extraversion and social anxiety. Comput Hum Behav 2016 Aug;61:113.

[33] Ghosh A, Dasgupta S. Psychological predictors of Facebook use. J Indian Acad Appl Psychol 2015;41(1):101-109.

[35]Baker JR, Moore SM. Distress, coping, and blogging: comparing new Myspace users by their intention to blog. Cyberpsychol Behav 2008 Feb;11(1):81-85.

[36]Baker JR, Moore SM. Blogging as a social tool: a psychosocial examination of the effects of blogging. Cyberpsychol Behav 2008 Dec;11(6):747-749.

[37]Deters FG, Mehl MR. Does posting Facebook status updates increase or decrease loneliness? An online social networking experiment. Soc Psychol Pers Sci 2013 Sep 1;4(5)

[38]Szwedo DE, Mikami AY, Allen JP. Qualities of peer relations on social networking websites: predictions from negative mother-teen interactions. J Res Adolesc 2011 Sep;21(3):595-607

[39] Casale S, Fioravanti G. Satisfying needs through social networking sites: a pathway towards problematic Internet use for socially anxious people? Addictive Behav Rep 2015 Jun;1:34-39. [CrossRef]

[40]Burke TJ, Ruppel EK. Facebook self-presentational motives: daily effects on social anxiety and interaction success. Commun Stud 2015;66(2):204-217.

[41]Bodroža B, Jovanović T. Validation of the new scale for measuring behaviors of Facebook users: psychoSocial Aspects of Facebook Use (PSAFU). Comput Hum Behav 2016 Jan;54:425-435.

[42]Landoll RR, La Greca AM, Lai BS. Aversive peer experiences on social networking sites: development of the Social Networking-Peer Experiences Questionnaire (SN-PEQ). J Res Adolesc 2013 Dec $1 ; 23(4)$

[43]Moberg FB, Anestis MD. A preliminary examination of the relationship between social networking interactions, internet use, and thwarted belongingness. Crisis 2015 May;36(3):187-193.

[44]Hong J, Hwang M, Hsu C, Tai K, Kuo Y. Belief in dangerous virtual communities as a predictor of continuance intention mediated by general and online social anxiety: the Facebook perspective. Comput Hum Behav 2015 Jul;48:663-670.

[45]Frison E, Eggermont $S$. The impact of daily stress on adolescents' depressed mood: the role of social support 
seeking through Facebook. Comput Hum Behav 2015 Mar;44:315-325.

[46] Ophir, Yaakov. "SOS on SNS: Adolescent distress on social network sites." Computers in Human Behavior 68 (2017): 51-55.

[47] Dang, Van Thac. "Information confusion and intention to stop using social networking site: a moderated mediation study of psychological distress and perceived novelty." Information Technology \& People (2020).

[48]Ophir, Yaakov. "SOS on SNS: Adolescent distress on social network sites." Computers in Human Behavior 68 (2017): 51-55.

[49]Bazarova, Natalya N., Yoon Hyung Choi, Janis Whitlock, Dan Cosley, and Victoria Sosik. "Psychological distress and emotional expression on Facebook." Cyberpsychology, Behavior, and Social Networking 20, no. 3 (2017): 157-163.

[50]Marino, Claudia, Gianluca Gini, Alessio Vieno, and Marcantonio M. Spada. "The associations between

problematic Facebook use, psychological distress and wellbeing among adolescents and young adults: A systematic review and meta-analysis." Journal of Affective Disorders 226 (2018): 274-281

[51]Stronge, Samantha, Tara Mok, Anastasia Ejova, Carol Lee, Elena Zubielevitch, Kumar Yogeeswaran, Diala Hawi, Danny Osborne, Joseph Bulbulia, and Chris G. Sibley. "Social media use is (weakly) related to psychological distress." Cyberpsychology, Behavior, and Social Networking 22, no. 9 (2019): 604-609.

[52]Tsai C, Shen P, Chiang Y. Meeting ex-partners on Facebook: users' anxiety and severity of depression. Behav Inf Technol 2015;34(7):668-677

[53]Settanni M, Marengo D. Sharing feelings online: studying emotional well-being via automated text analysis of Facebook posts. Front Psychol 2015;6:1045

[54]Dumitrache SD, Mitrofan L, Petrov Z. Self-image and depressive tendencies among adolescent Facebook users. Revista de Psihologie 2012;58(4):285-295 